\title{
Combined Index of Hemoglobin, Albumin, Lymphocyte, and Platelet (HALP) Predict Recurrence After Radical Resection in Gastrointestinal Stromal Tumors: a propensity scores matching analysis
}

\author{
Zhou Zhao ${ }^{1}$, Xiaonan Yin ${ }^{1}$, Jian Wang ${ }^{1}$, Xin Chen $^{1}$, Zhaolun Cai ${ }^{1}$, and Bo Zhang ${ }^{1}$ \\ ${ }^{1}$ Sichuan University West China Hospital
}

June 20, 2021

\begin{abstract}
The combined index of hemoglobin, albumin, lymphocyte, and platelet (HALP) can reflect systemic inflammation and nutritional status simultaneously, with some evidence revealing its prognostic value for some tumors. However, the effect of HALP on recurrence-free survival (RFS) in patients of gastrointestinal stromal tumors (GISTs) has not been reported. Therefore, the present study aimed to investigate the prognostic value of HALP in GIST patients. Methods Data from 591 untreated patients who underwent R0 resection for primary and localized GISTs at West China Hospital between December 2008 and December 2016 were included. Clinicopathological data, preoperative albumin, blood routine information, postoperative treatment, and recurrence status were recorded. To eliminate the baseline inequivalence, the propensity scores matching (PSM) method was introduced. The relationship between RFS and preoperative HALP was investigated. Results The optimal cutoff value for the HALP was determined by the $\mathrm{x}$-Tile analysis at 31.5. HALP was significantly associated with tumor site, tumor size, mitosis, Ki67, NIH risk category and adjuvant therapy (all P<0.001). Before PSM, GIST patients with an increased HALP had a significantly poor RFS $(\mathrm{P}<0.001)$, and low HALP was an independent risk factor for poor RFS (HR=0.0551, 95\% CI: 0.313 - 0.968, $\mathrm{P}=0.038)$. In NIH high-risk GIST patients, GIST patients with low HALP had a worse RFS than patients with high HALP $(\mathrm{P}<0.05)$. After PSM, 188 pairs of GIST patients were identified, GIST patients with an increased HALP still had a significantly poor RFS after PSM $(\mathrm{P}<0.001)$, and low HALP was still an independent risk factor for poor RFS $(\mathrm{HR}=0.585,95 \%$ CI: $0.316-0.972, \mathrm{P}=0.042)$. Conclusions HALP had a statistically significant correlation with postoperative pathology and postoperative treatment. Furthermore, HALP has a strong ability to predict the RFS in GIST patients with radical resection.
\end{abstract}

Combined Index of Hemoglobin, Albumin, Lymphocyte, and Platelet (HALP) Predict Recurrence After Radical Resection in Gastrointestinal Stromal Tumors: a propensity scores matching analysis

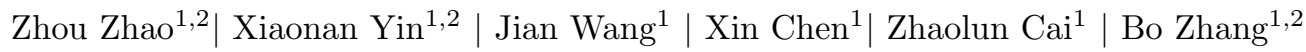

${ }^{1}$ Department of Gastrointestinal Surgery, Sichuan University West China Hospital, Chengdu, China

${ }^{2}$ Department of General Surgery: Research Laboratory of Tumors Epigenetics and Genomics, Sichuan University West China Hospital, Chengdu, China.

\section{Corresponding Author}

Bo Zhang, MD, Department of Gastrointestinal Surgery, West China Hospital, Sichuan University, No. 37 Guoxue Alley, Chengdu, China. Fax number: +86-28-85422872; E-mail address:hxwcwk@126.com. 


\section{ABSTRACT}

BackgroundThe combined index of hemoglobin, albumin, lymphocyte, and platelet (HALP) can reflect systemic inflammation and nutritional status simultaneously, with some evidence revealing its prognostic value for some tumors. However, the effect of HALP on recurrence-free survival (RFS) in patients of gastrointestinal stromal tumors (GISTs) has not been reported. Therefore, the present study aimed to investigate the prognostic value of HALP in GIST patients.

Methods Data from 591 untreated patients who underwent R0 resection for primary and localized GISTs at West China Hospital between December 2008 and December 2016 were included. Clinicopathological data, preoperative albumin, blood routine information, postoperative treatment, and recurrence status were recorded. To eliminate the baseline inequivalence, the propensity scores matching (PSM) method was introduced. The relationship between RFS and preoperative HALP was investigated.

Results The optimal cutoff value for the HALP was determined by the x-Tile analysis at 31.5. HALP was significantly associated with tumor site, tumor size, mitosis, Ki67, NIH risk category and adjuvant therapy (all $\mathrm{P}<0.001)$. Before PSM, GIST patients with an increased HALP had a significantly poor RFS $(\mathrm{P}<$ 0.001), and low HALP was an independent risk factor for poor RFS (HR=0.0551, 95\% CI: 0.313 - 0.968, $\mathrm{P}=0.038$ ). In NIH high-risk GIST patients, GIST patients with low HALP had a worse RFS than patients with high HALP $(\mathrm{P}<0.05)$. After PSM, 188 pairs of GIST patients were identified, GIST patients with an increased HALP still had a significantly poor RFS after PSM $(\mathrm{P}<0.001)$, and low HALP was still an independent risk factor for poor $\mathrm{RFS}(\mathrm{HR}=0.585,95 \% \mathrm{CI}$ : $0.316-0.972, \mathrm{P}=0.042)$.

Conclusions HALP had a statistically significant correlation with postoperative pathology and postoperative treatment. Furthermore, HALP has a strong ability to predict the RFS in GIST patients with radical resection.

Key Words: gastrointestinal stromal tumors (GISTs); Combined index of hemoglobin, albumin, lymphocyte, and platelet (HALP); recurrence-free survival (RFS).

\section{What's known?}

The comprehensive index of hemoglobin, albumin, lymphocytes, and platelets (HALP) can indicate both systemic inflammation and nutritional status. Some evidence suggested that it has prognostic value for certain tumors, but excluded gastrointestinal stromal tumors.

\section{What's new?}

HALP had a statistically significant correlation with postoperative pathology and postoperative treatment in GIST. Furthermore, HALP has a strong ability to predict the RFS in GIST patients with radical resection.

\section{INTRODUCTION}

Gastrointestinal stromal tumors (GISTs), a rare type of tumor, are the most frequent mesenchymal tumors arising from gastrointestinal tract ${ }^{1}$. GISTs may occur anywhere in the digestive tract and even outside the gastrointestinal tract occasionally, with the stomach accounting for $60 \%$ and the small intestine $30 \%{ }^{2}$. The morphology, immunohistochemistry, and molecular markers are helpful to the diagnosis of GISTs. Surgical resection is the standard treatment for resectable GISTs ${ }^{3}$. Nowadays, novel small molecular tyrosine kinase inhibitors, such as imatinib and sunitinib, have revolutionized the integrated treatment of GISTs and greatly improved the long-term prognosis of patients ${ }^{4}$.

Currently, some GIST-specific parameters based on postoperative pathologies, such as tumor size, primary tumor location, mitotic index, and tumor rupture, have been used to stratify the risk of recurrence for GISTs $^{2,5-7}$. Meanwhile, the recent effort has shed light on the role of preoperative cancer-related inflammation and nutrition status in cancer progression, such as gastric cancer ${ }^{8}$, colorectal cancer ${ }^{9}$, non-small lung 
cancer $^{10}$, and gastrointestinal stromal tumor ${ }^{11-16}$. Several preoperative immuno-inflammatory-based prognostic scores, such as the preoperative neutrophil to lymphocyte ratio (NLR), the lymphocyte to monocyte ratio (LMR), and the platelet to lymphocyte ratio (PLR), reflect the systematic inflammatory response, with some evidence revealing that they are prognostic for GISTs ${ }^{13-17}$. Furthermore, nutritional status, such as the prognostic nutritional index (PNI), has also been shown to play an important role in GIST progression ${ }^{10,11}$.

Recent studies have proposed a new combined index of hemoglobin, albumin, lymphocyte, and platelet (HALP), which is composed of hemoglobin, albumin, lymphocytes and platelets, and can reflect systemic inflammation and nutritional status simultaneously ${ }^{18}$. It has been reported to be related to the prognosis of patients with pancreatic cancer ${ }^{19}$, renal cancer ${ }^{20}$, gastric cancer ${ }^{18}$, prostate cancer ${ }^{21}$, bladder cancer ${ }^{22}$, esophageal cancer ${ }^{23}$ and small cell lung cancer ${ }^{24}$. However, there are no studies on the relationship between HALP and recurrence in GIST patients with radical resection. Therefore, this study aimed to investigate the prognostic value of preoperative HALP in resected GIST patients.

\section{METHOD}

\section{Patient population}

A flow diagram of the patient selection process is shown inFigure 1. Data from consecutive, previously untreated patients who underwent $\mathrm{R} 0$ resection for primary, localized GISTs at West China Hospital between December 2008 and December 2016 were included in this study. Patients younger than 18 years, without complete preoperative blood routine information or medical history, with infectious diseases, WBC $>10 \times$ $10^{\wedge} 9 / \mathrm{L}$, neutrophils $>8 \times 10^{\wedge} 9 / \mathrm{L}$, or lymphocyte $>5 \times 10^{\wedge} 9 / \mathrm{L}$, with other tumors, with severe liver, kidney, or heart diseases, with emergency surgery, and follow-up less than 6 months were excluded. Finally, 591 GIST patients were enrolled for the current analysis.

This study was reviewed and approved by the Ethics Committee of the West China Hospital of Sichuan University. All patients provided written informed consent.

\section{Definition}

RFS was defined as the time interval between the time of surgery and the time of the first documented appearance of tumor after complete resection. The HALP, PNI, NLR, PLR, and LMR were calculated using the following formulas: hemoglobin level $(\mathrm{g} / \mathrm{L}) \times$ albumin level $(\mathrm{g} / \mathrm{L}) \times$ lymphocyte count $(/ \mathrm{L}) /$ platelet count $(/ \mathrm{L})^{19}$, albumin level $(\mathrm{g} / \mathrm{L})+5 \times$ lymphocyte count $\left(\text { number } / \mathrm{mm}^{3}\right)^{25}$, neutrophil count (number $/ \mathrm{mm}^{3}$ )/ lymphocyte count (number $\left./ \mathrm{mm}^{3}\right)^{15,16}$, platelet count (number $/ \mathrm{mm}^{3}$ ) / lymphocyte count (number $\left./ \mathrm{mm}^{3}\right)^{14}$, lymphocyte count (number $/ \mathrm{mm}^{3}$ ) / monocyte count (number $\left./ \mathrm{mm}^{3}\right)^{26}$, respectively.

\section{Data collection}

Clinicopathological data, postoperative treatment, and recurrence status were recorded. The following data of each patient were retrieved from the self-built GISTs database: demographic characteristics, tumor sites, tumor size, mitotic index (mitosis / 50 high power field or mitosis / $50 \mathrm{~mm}^{2}$ ), morphology, immunohistochemistry, molecular markers, preoperative hemoglobin, albumin, white blood cells count, absolute neutrophil count, monocyte count, platelet count, and lymphocyte count. Tumor risk stratification was determined based on "the modified National Institutes of Health (NIH) classification" ${ }^{27}$.

\section{Perioperative Evaluation and Follow-up}

The laboratory tests were evaluated within 1 week before operation. The parameters included complete blood cell count and serum albumin. Abdominal ultrasonography or computed tomography was performed every 3 - 6 months in the first 3 years after operation, and then every 6 - 12 months until 5 years after the operation, and then once a year until recurrence. The recurrence status of patients was ascertained by December 2020. 


\section{Statistical analysis}

The optimal cutoff values for the HALP, PNI, NLR, PLR, and LMR were determined by the $\mathrm{x}$-Tile analysis at 31.5, 48.6, 2.60, 134.8, and 4.0, respectively ${ }^{28}$. PSM was performed as 1:1 matching with nearest neighbor matching and a 0.1 caliper based on the patient's age, tumor size, tumor site, mitosis, Ki67, intratumoral hemorrhage, intratumoral necrosis and postoperative targeted therapy using nearest neighbor matching with MatchIt $\mathrm{R}$ package. The categorical variables are reported as numbers (\%) and quantitative variables are reported as the means $\pm \mathrm{SD}$ or medians (range). Statistical significance of group comparisons was analyzed via parametric and nonparametric tests for continuous variables and via chi-square analysis or Fisher test for categorical variables. Survival curves of the RFS were calculated by the Kaplan-Meier methods and compared by log-rank tests. Hazard ratios for recurrence were calculated by Cox regression analysis. Sensitivity and specificity HALP, PNI, NLR, LMR, and PLR were defined using time dependent receiver operating characteristics (ROC) curves, and areas under the curve (AUC) were detected utilizing survivalROC R package ${ }^{29}$. All statistical analyses were performed using SPSS Statistics version 21 (SPSS 21.0; SPSS Inc) and GraphPad Prism version 7.0 (GraphPad Software). Statistical significance was set at P $<0.05$ as two-sided.

\section{RESULTS}

\section{Baseline characteristics}

The demographic and clinicopathological characteristics of the 591 GIST patients were listed in Table 1 . The study population consisted of $280(46.8 \%)$ male and 311 (53.2\%) female patients. The median (range) age was $57(21-86)$ years. The median follow-up time was 56 months (range, 4-138). The means \pm SD for the HALP, the PNI, the NLR, the PLR, and the LMR values were $45.81 \pm 33.73,49.04 \pm 5.43,2.64 \pm 1.74,152.8$ \pm 84.6 and $5.13 \pm 3.00$, respectively. The means $\pm \mathrm{SD}$ of tumor size was $6.16 \pm 4.87 \mathrm{~cm} .191$ tumors $(32.3 \%)$ had a mitotic index of $>5 / 50$ high-power field. A total of $34.0 \%(201 / 691)$ of the GIST patients received adjuvant therapy with imatinib or sunitinib. According to NIH risk classification, $72(12.2 \%)$ patients were classified as very low risk, $178(30.1 \%)$ patients were classified as low risk, 114 (19.3\%) patients were classified as intermediate risk, and $227(38.4 \%)$ patients were classified as high risk. Recurrence occurred in 62 GIST patients.

\section{Association of HALP and clinicopathological factors}

The clinicopathological characteristics between the high and low groups of HALP were categorized and analyzed in Table 1 . Together, 229 patients were assigned to the low HALP group and 362 patients to the high HALP group. The results demonstrated that tumor site, tumor size, mitotic index, Ki67, intratumoral hemorrhage, intratumoral necrosis, NIH risk category, and adjuvant therapy were associated with HALP (all $\mathrm{p}<0.05)$.

PSM analysis was further carried out to avoid confounding variables that might interfere with the association between RFS and HALP level. After 1:1 matching, PSM analysis identified 188 pairs of GIST patients. After PSM, HALP were still associated with gender, histologic subtypes, NLR, PLR, LMR, and PNI, but not with other clinicopathological characteristics (Table 1 ).

\section{Association of clinicopathological factors and RFS}

Before PSM, tumor site, tumor size, mitotic index, Ki67, intratumoral hemorrhage, intratumoral necrosis, NIH risk category, albumin, neutrophils, platelets, NLR, PLR, PNI, and HALP were associated with RFS (all $\mathrm{P}<0.05$ ) (Table 2 ). RFS in GIST patients with low HALP were significantly poor than patients with high HALP (Figure 2 ). Cox multiple regression analysis showed that HALP was an independent prognostic factor for RFS in GIST patients before PSM ( $\mathrm{HR}=0.506,95 \%$ CI: 0.291-0.879, $\mathrm{P}=0.016)$. 
After PSM, tumor site, tumor size, mitotic index, Ki67, intratumoral hemorrhage, intratumoral necrosis, NIH risk category, albumin, neutrophils, PNI, and HALP were still related to RFS (all P < 0.05) (Table 2 ). RFS was also significantly poor in GIST patients with low HALP than patients with high HALP. (Figure 2 ). Furthermore, Cox multiple regression analysis showed that HALP was an independent prognostic factor for RFS in GIST patients (HR=0.585, 95\% CI: $0.316-0.972, \mathrm{P}=0.042)$.

\section{Subgroup analysis}

The clinicopathological characteristics of high-risk GIST patients between the high and low groups of HALP were categorized in Table S1. Together, 125 patients were assigned to the low HALP group and 102 patients to the high HALP group. The results demonstrated that gender, Ki67, intratumoral hemorrhage, intratumoral necrosis were associated with HALP (all $\mathrm{p}<0.05$ ). Not surprisingly, patients in the low HALP group had significantly worse survival than patients in the high HALP group (Figure 2 ). Furthermore, Cox multiple regression analysis indicated that HALP was an independent prognostic factor for RFS in GIST patients $(\mathrm{HR}=0.469,95 \%$ CI: 0.245-0.896, $\mathrm{P}=0.022)$ (Table S2 ).

\section{Sensitivity analysis}

As sensitivity analysis, time dependent ROC was generated for HALP, PNI, NLR, LMR, and PLR to predict five-year RFS. According to the results, HALP had the highest sensitivity and accuracy $(\mathrm{AUC}=0.661$ ) in predicting five-year RFS, while the AUC of PNI, NLR, LMR, and PLR were 0.622, 0.591, 0.505, and 0.627, respectively (Figure 3 ).

In addition, to assess consistency of HALP prediction, Cox multiple regression analysis in GIST patients before PSM, after PSM and in high-risk subgroups were performed to better assess the impact of each type of covariates on the association between HALP and RFS. In the three models, tumor site, mitotic index, Ki67, adjuvant therapy, and HALP were all proved as independent prognostic factors for poor RFS, but tumor size was not in high-risk GIST patients (Table 2 \& Table S2 ).

\section{DISCUSSION}

There is growing evidence that preoperative nutritional status and inflammatory response may be a potentially powerful predictor of the prognosis of cancer patients. Consistent with previous research, preoperative inflammation scores, such as NLR and PLR, were associated with the prognosis of GIST patients before PSM and after PSM in the present study ${ }^{14,16,30,31}$. However, LMR seemed to be irrelevant to the RFS of GIST patients, which is different from previous studies ${ }^{14}$. In addition, the PNI, a nutritional score based on albumin levels and lymphocytes, was also related to RFS of GIST patients before PSM and after PSM in present study ${ }^{11,12}$ (Figure S1).

In this study, we found that preoperative HALP was significantly correlated with tumor site, tumor size, mitosis, Ki67, intratumoral hemorrhage, intratumoral necrosis, NIH risk category and adjuvant therapy. Anemia is one of the most common symptoms of GIST, which may be caused by gastrointestinal bleeding and intratumoral bleeding 32 . Additionally, since tumor cells synthesize proteins with albumin, this will result in hypoalbuminemia in GIST patients. As a result, it is unsurprising that HALP, which is composed of hemoglobin and albumin, is associated with parameters indicating the degree of malignancy in GIST.

To avoid the impact of these biases on RFS, we utilized the PSM method to balance tumor site, tumor size, mitosis, Ki67, intratumoral hemorrhage, intratumoral necrosis, and adjuvant therapy. After PSM, gender, histologic subtypes, PNI, NLR, LMR, and PLR were still associated with HALP. Importantly, there were no difference in risk factors (tumor site, tumor size, mitosis, Ki67, NIH risk category, and adjuvant therapy) in the low/high HALP group. Given that HALP shared the same parameters with PNI, NLR, LMR, and PLR, their statistically significant correlation is unsurprising. The correlation between HALP and gender mainly attributed to the difference of hemoglobin level between male and female patients $(123.22 \pm 2.08 \mathrm{~g} / \mathrm{L}$ for male and $105.46 \pm 1.84 \mathrm{~g} / \mathrm{L}$ for female, $\mathrm{P}<0.001)$. Additionally, the correlation between HALP and histologic 
subtypes mainly attributed to insufficient sample size in epithelioid subgroup, where the patients of low HALP group was 0 , while patient of the high HALP group was eight. Notably, neither gender nor histologic subtype was associated with recurrence ( Table $S 1$ ). Subgroup analysis based on gender demonstrated that a low level of HALP was associated with recurrence in female patients but not in male patients. However, there was still a trend of poor prognosis in male patients with lower HALP (Figure S2 ). The reason for this phenomenon might be the insufficient sample size, but further research was needed.

Finally, consistent with previous research on HALP in other tumor ${ }^{18-24}$, our findings revealed prognostic value of HALP in GIST. HALP was an independent risk factor for GIST patients before PSM, after PSM, and in high-risk subgroups. GIST patients with low HALP before PSM, after PSM, and in high-risk subgroups all had a poor prognosis. Thus, HALP can be used not only to evaluate the postoperative risk classification of GIST patients prior to surgery, but also to assess their prognosis. Notably, the HALP index can be conveniently and inexpensively applied to predict the prognosis of patients.

Although the underlying mechanism of systemic inflammation in tumorigenesis, progression and metastasis remains obscure, there are some theories that it stimulates angiogenesis, immunosuppression and the formation of supporting microenvironment. It is well known that lymphocytes play an important role in inhibiting tumor growth ${ }^{33-35}$. A higher lymphocyte signature was associated with improved prognosis in a variety of tumors $^{35}$. Whereas, platelets could infiltrate into the tumor microenvironment and interact with cancer cells directly, helping circulating tumor cell attach to endothelial cells and providing signals to establish a niche environment before metastasis ${ }^{36-42}$

Zheng-Yang Yang et al. found that GIST with gastrointestinal bleeding was independent prognostic predictors for poor RFS ${ }^{43}$. Some studies have shown that low hemoglobin can lead to tumor hypoxia, which has a higher risk of local failure and distant metastasis ${ }^{31,44}$. Furthermore, a hypoxic tumor environment could induce limited accumulation of drugs and hinder the efficacy of drugs ${ }^{45}$. Additionally, one of the primary adverse effects of imatinib is anemia ${ }^{46}$, which may be prevented by a normal preoperative hemoglobin levels, thus improving imatinib treatment compliance.

Low levels of serum albumin were also associated with poor long-term survival in GIST patients ${ }^{44,45}$, which was consistent with our findings. Serum albumin is generally considered to be associated with nutritional status and liver or renal function, both of which may affect patients' compliance with imatinib therapy, similar to hemoglobin. Moreover, about $95 \%$ of imatinib is bound to serum proteins, mainly albumin and 1 -acid glycoprotein ${ }^{47}$. Tumors tissues have abnormal vascular endothelial gaps and lack effective lymphatic drainage, allowing macromolecules more likely to accumulate in the tumor tissue than normal tissue ${ }^{48,49}$. This effect is referred to as the enhanced permeability and retention effect. Albumin exerts this effect as a result of its unique molecular size, which may facilitate drug accumulation in tumors and improve therapeutic effect ${ }^{50}$.

There are some limitations to this study. Firstly, this study is a retrospective study, so there may be biases in the process of data collection. Secondly, our cases were collected from 2008 to 2016, during which time imatinib has been used in the adjuvant treatment of GIST in China. Despite the adverse reaction and higher costs, 201/591(34.0\%) of GIST patients still received adjuvant imatinib therapy. As an important treatment after GIST, adjuvant imatinib therapy can significantly improve the prognosis of GIST patients ${ }^{51}$, and its benefits are also shown in present study. However, there was no adequate collection and analysis of the time, dose and adverse reactions of patients with imatinib or sunitinib therapy, which may be also related to HALP. Moreover, this study also did not evaluate other clinicopathological factors, especially gene mutation status, which also relate to prognosis. Most importantly, nutritional status may be associated with the economic status, which is a critical factor influencing medication compliance and prognosis. Furthermore, the effect of preoperative or postoperative improvement of nutritional status or inflammation response on the prognosis of GIST remained obscure, which needed to be further confirmed in clinical studies. 


\section{CONCLUSION}

A low level of HALP was related to tumor site, tumor size, mitosis, Ki67, NIH risk category and adjuvant

therapy. A low level of HALP was considered to be an important risk factor for RFS in GIST patients with $\mathrm{R} 0$ resection.

\section{FUNDING INFORMATION}

This study was funded by the National Natural Science Foundation of China (Grant No. 81572931) and 1.3.5 project for disciplines of excellence, West China Hospital, Sichuan University (ZYJC18034).

\section{CONFLICT OF INTEREST}

None declared.

\section{AUTHOR CONTRIBUTION}

Zhou Zhao: wrote the manuscript and followed up.

Xiaonan Yin: collected the clinicopathological data.

Jian Wang: performed statistical analysis.

Xin Chen: collected the clinicopathological data and followed up.

Zhaolun Cai: supervised the resolved disputes in the data collection.

Bo Zhang: supervised and revised the report

Table 1. Baseline characteristics in patients with high or low HALP before and after PSM.

\begin{tabular}{|c|c|c|}
\hline \multirow[t]{2}{*}{ Characteristics } & Before PSM & Before PSM \\
\hline & All & Low HALP $(<31.5)$ \\
\hline $\mathrm{n}(\%)$ & 591 & $229(38.7)$ \\
\hline Age (yrs, mean \pm SD) & $56.3 \pm 12.0$ & $56.7 \pm 12.2$ \\
\hline$<60$ & $337(57.0)$ & 129 \\
\hline 60 & $254(43.0)$ & 100 \\
\hline \multicolumn{3}{|l|}{ Gender } \\
\hline Male & $280(47.4)$ & 98 \\
\hline Female & $311(52.6)$ & 131 \\
\hline \multicolumn{3}{|l|}{ Tumor site } \\
\hline Stomach & $424(71.7)$ & 143 \\
\hline Non-stomach & $167(28.3)$ & 86 \\
\hline Tumor size $(\mathrm{cm}$, mean $\pm \mathrm{SD})$ & $6.16 \pm 4.87$ & $7.69 \pm 5.65$ \\
\hline 2 & $86(14.6)$ & 10 \\
\hline $2.1-5.0$ & $251(42.5)$ & 87 \\
\hline $5.1-10.0$ & $184(31.1)$ & 95 \\
\hline$>10.0$ & $70(11.8)$ & 37 \\
\hline \multicolumn{3}{|l|}{ Mitotic index /50HPF } \\
\hline 5 & $332(56.2)$ & 107 \\
\hline $6-10$ & $100(16.9)$ & 45 \\
\hline$>10$ & $91(15.4)$ & 49 \\
\hline
\end{tabular}




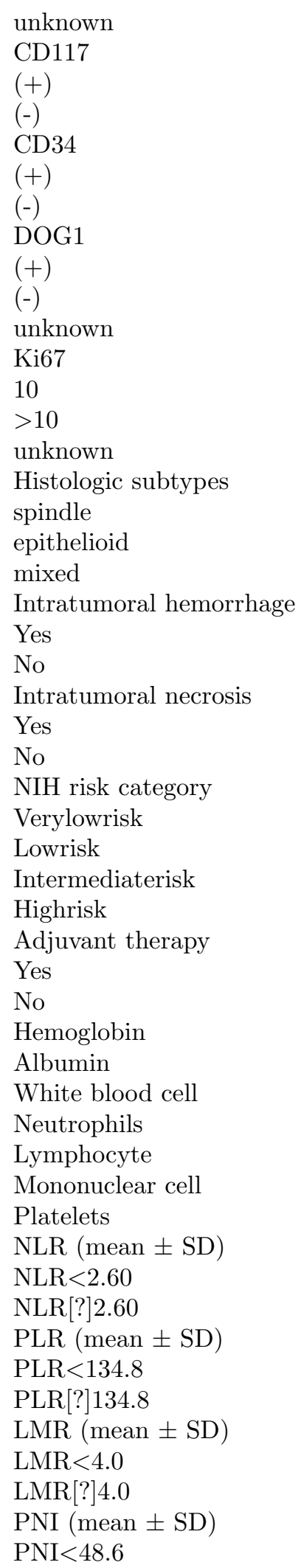

$573(97.0)$

$18(3.0)$

$527(89.2)$

64 (10.8)

201

28

$529(89.5)$

211

$10(1.7)$

$52(8.8)$

$417(70.6) \quad 140$

$98(16.6)$

$76(12.9)$

61

28

$518(87.6) \quad 197$

$13(2.2)$

$60(10.2)$

3

29

108 (18.3)

$483(81.7)$

64

165

117 (19.8)

474 (80.2)

71

158

$72(12.2)$

$178(30.1)$

$114(19.3)$

227 (38.4)

9

52

43

125

201 (34.0)

99

$390(66.0)$

130

$118.30 \pm 26.80$

$94.60 \pm 22.81$

$39.52 \pm 4.40$

$5.32 \pm 1.55$

$3.60 \pm 1.40$

$1.21 \pm 0.42$

$0.33 \pm 0.17$

$261.6 \pm 95.7$

$3.41 \pm 2.36$

99

130

$228.7 \pm 84.1$

12

217

$4.45 \pm 3.05$

110

119

$45.6 \pm 5.03$

171 
PNI[?]48.6

Recurrence

Yes

No

${ }^{1}$ Method $=$ nereast Cliper value $=0.1$

$\mathrm{P}<0.05$ was considered statistically significant.
$321(54.3)$

$62(10.5)$

$529(89.5)$

${ }^{1}$ Method $=$ nereast; Cliper value $=0.1$

${ }^{*} \mathrm{P}<0.05$ was considered statistically significant.
58

42

187

${ }^{1}$ Method $=$ nereast; $\mathrm{C}$

Table 2. Univariate and multivariate regression analysis of prognostic factors in patients before and after PSM.

Risk factors

Age

Gender (male vs female)

Tumor site (stomach vs non-stomach)

Tumor size $(\mathrm{cm})([?] 2 / 2.1-5.0 / 5.1-10.0 />10.0)$

Mitotic index (/50HPF) ([?]5 / 6-10 / >10 / unknown)

5 vs $6-10$

5 vs $>10$

5 vs unknown

CD117 (+ / - )

CD34 $(+/-)$

DOG1 $(+/-/$ unknown $)$

Ki67 ([?]10 / >10 / unknown)

$>10$ vs [?]10

unknown vs [?]10

Histologic subtypes (spindle / epithelioid / mixed)

Intratumoral hemorrhage (yes / no)

Intratumoral necrosis (yes / no)

NIH risk category (very low/low/intermediate/high)

Hemoglobin

Albumin

White blood cell

Neutrophils

Lymphocyte

Monocyte

Platelets

Adjuvant therapy (yes / no)

$\operatorname{NLR}(<2.60 /[?] 2.60)$

PLR $(<134.8 /[?] 134.8)$

$\operatorname{LMR}(<4.0 /[?] 4.0)$

PNI $(<48.6 /[?] 48.6)$

$\operatorname{HALP}(<31.5 /[?] 31.5)$

HR: Hazard ratio; CI: Confidence interval; NA: Not adopted; NS: Not significant. ${ }^{*} \mathrm{P}<0.05$ was considered statistically sig

Table S1. Demographic and clinicopathologic features of 227 resected high-risk GIST patients with high or low HALP. 


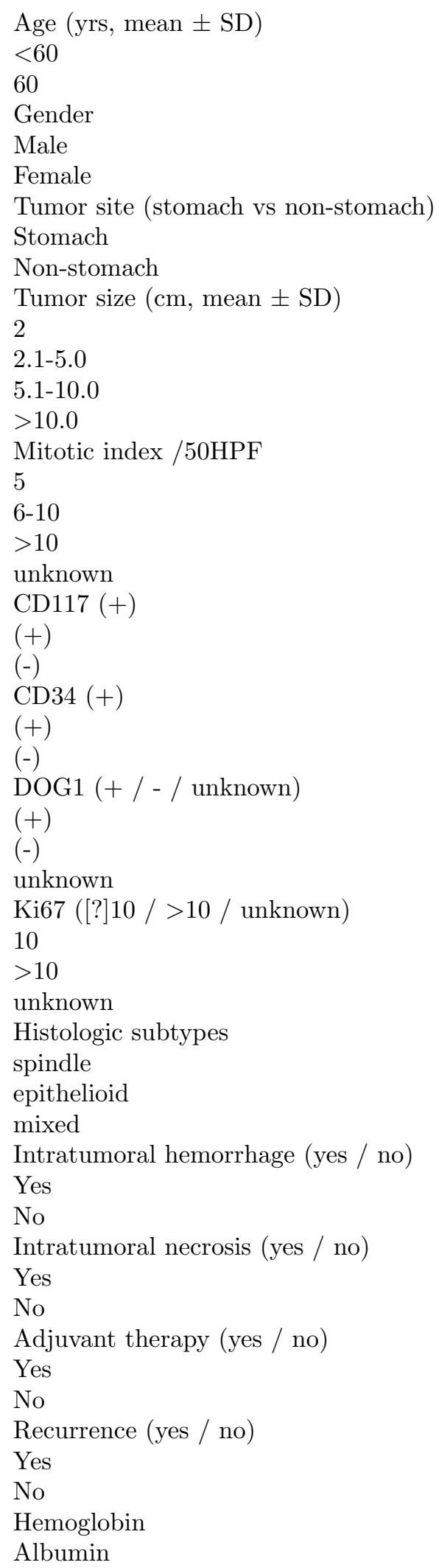


White blood cell

Neutrophils

Lymphocyte

Mononuclear cell

Platelets

NLR (mean $\pm \mathrm{SD})$

NLR $<2.60$

NLR[?]2.60

PLR (mean \pm SD)

PLR $<134.8$

PLR[?]134.8

$\mathrm{LMR}($ mean $\pm \mathrm{SD})$

$\mathrm{LMR}<4.0$

LMR[?]4.0

$\mathrm{PNI}($ mean $\pm \mathrm{SD})$

$\mathrm{PNI}<48.6$

PNI[?]48.6

$\operatorname{HALP}($ mean $\pm \mathrm{SD})$

HALP $<31.5$

HALP[?]31.5

$\mathrm{P}<0.05$ was considered statistically significant.
$5.63 \pm 1.46$

$3.65 \pm 1.21$

$1.44 \pm 0.55$

$0.35 \pm 0.15$

$222.8 \pm 96.3$

$2.96 \pm 2.09$

$123(54.2)$

104 (45.8)

$172.04 \pm 87.54$

88 (38.8)

$139(61.2)$

$4.88 \pm 3.69$

128 (56.4)

99 (43.6)

$47.31 \pm 5.50$

131 (57.7)

96 (42.3)

$37.41 \pm 30.15$

$125(55.1)$

102 (44.9)
$-$

$-$

52

73

6

119

61

64

96

29

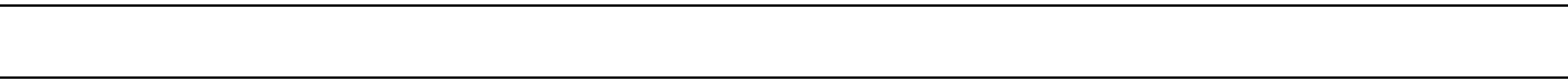

Table S2. Univariate and multivariate regression analysis of prognostic factors in high-risk patients

Characteristics

\begin{tabular}{c} 
Table \\
\hline Univar \\
HR (9) \\
1.011 \\
0.954 \\
1.035 \\
1.353 \\
1.968 \\
\\
\\
1.099 \\
0.705 \\
1.976 \\
1.959 \\
\\
1.024 \\
1.969 \\
2.086 \\
0.379 \\
1.784 \\
2.251 \\
0.925 \\
1.506 \\
0.430 \\
0.504 \\
\end{tabular}

Age

Gender

Tumor site (stomach vs non-stomach)

Tumor size $(\mathrm{cm})([?] 2 / 2.1-5.0 / 5.1-10.0 />10.0)$

Mitotic index (/50HPF) ([?]5 / 6-10 / >10 / unknown)

$6-10$ vs [?]5

$>10$ vs [?]5

unknown vs [?]5

CD117 $(+/-)$

CD34 $(+/-)$

DOG1 $(+/-/$ unknown $)$

$\operatorname{Ki67}([?] 10 />10 /$ unknown)

10 vs $>10$

10 vs unknown

Histologic subtypes (spindle / epithelioid / mixed)

Intratumoral hemorrhage (yes / no)

Intratumoral necrosis (yes / no)

Adjuvant therapy (yes / no)

NLR $(<2.60 /[?] 2.60)$

$\operatorname{PLR}(<134.8 /[?] 134.8)$

$\operatorname{LMR}(<4.0 /[?] 4.0)$

WHR $(<5.60 /[?] 5.60)$

PNI $(<48.6 /[?] 48.6)$

$\operatorname{HALP}(<31.5 /[?] 31.5)$

$\mathrm{P}<0.05$ was considered statistically significant. 


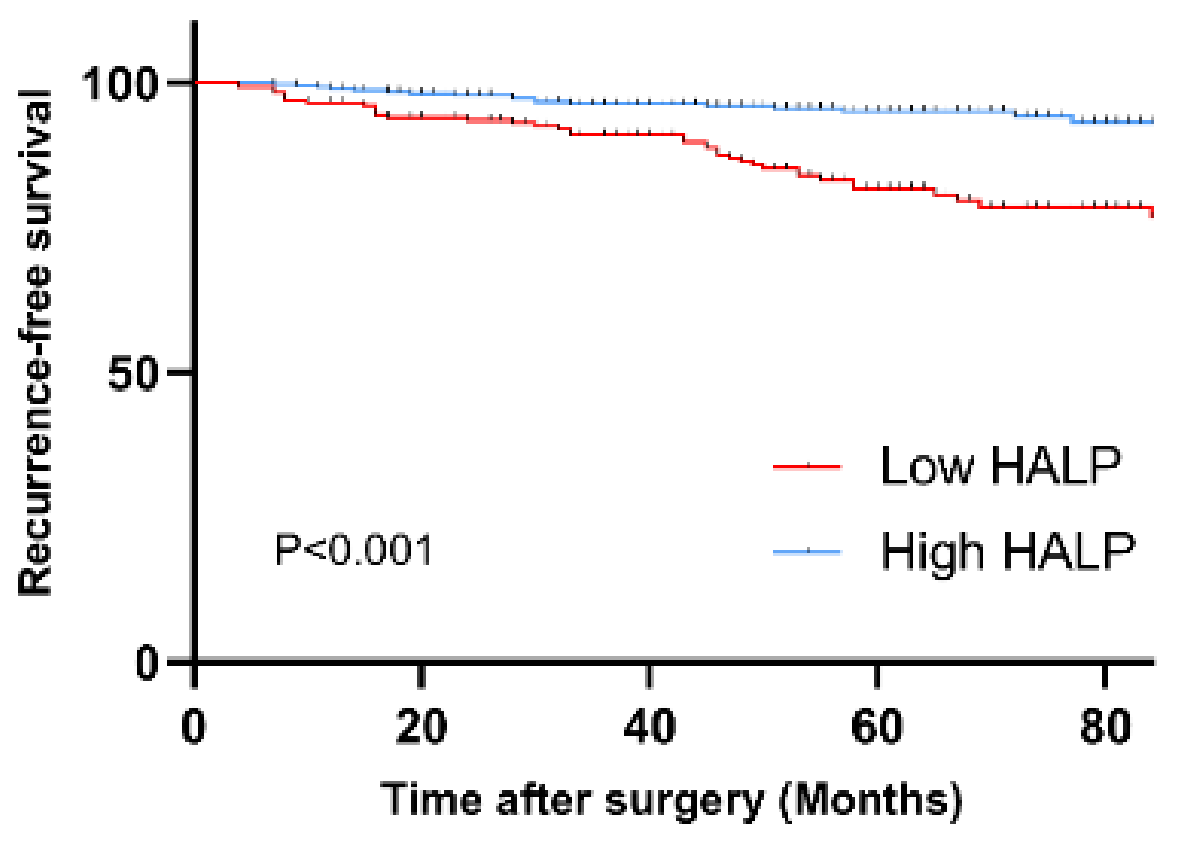

Time dependent ROC

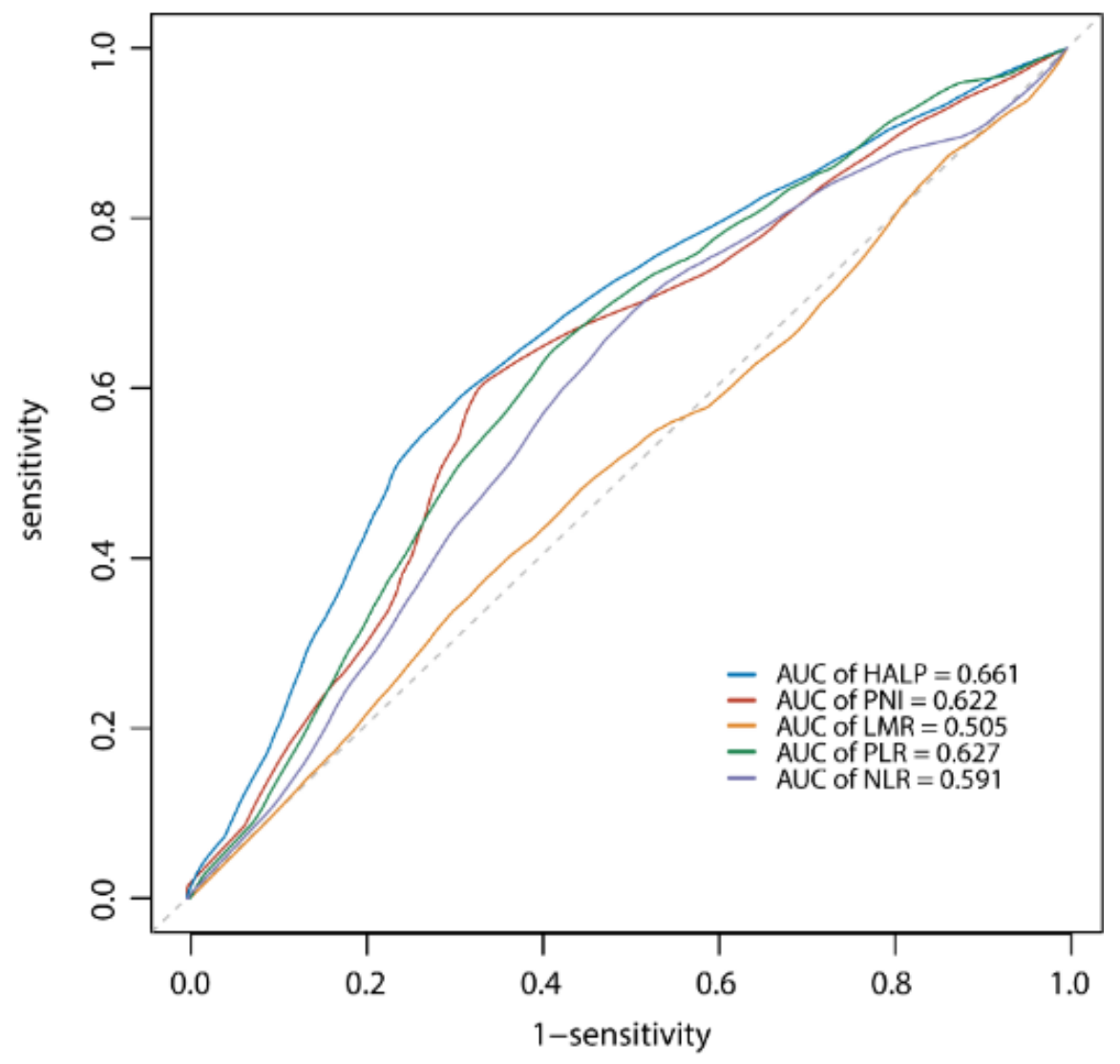



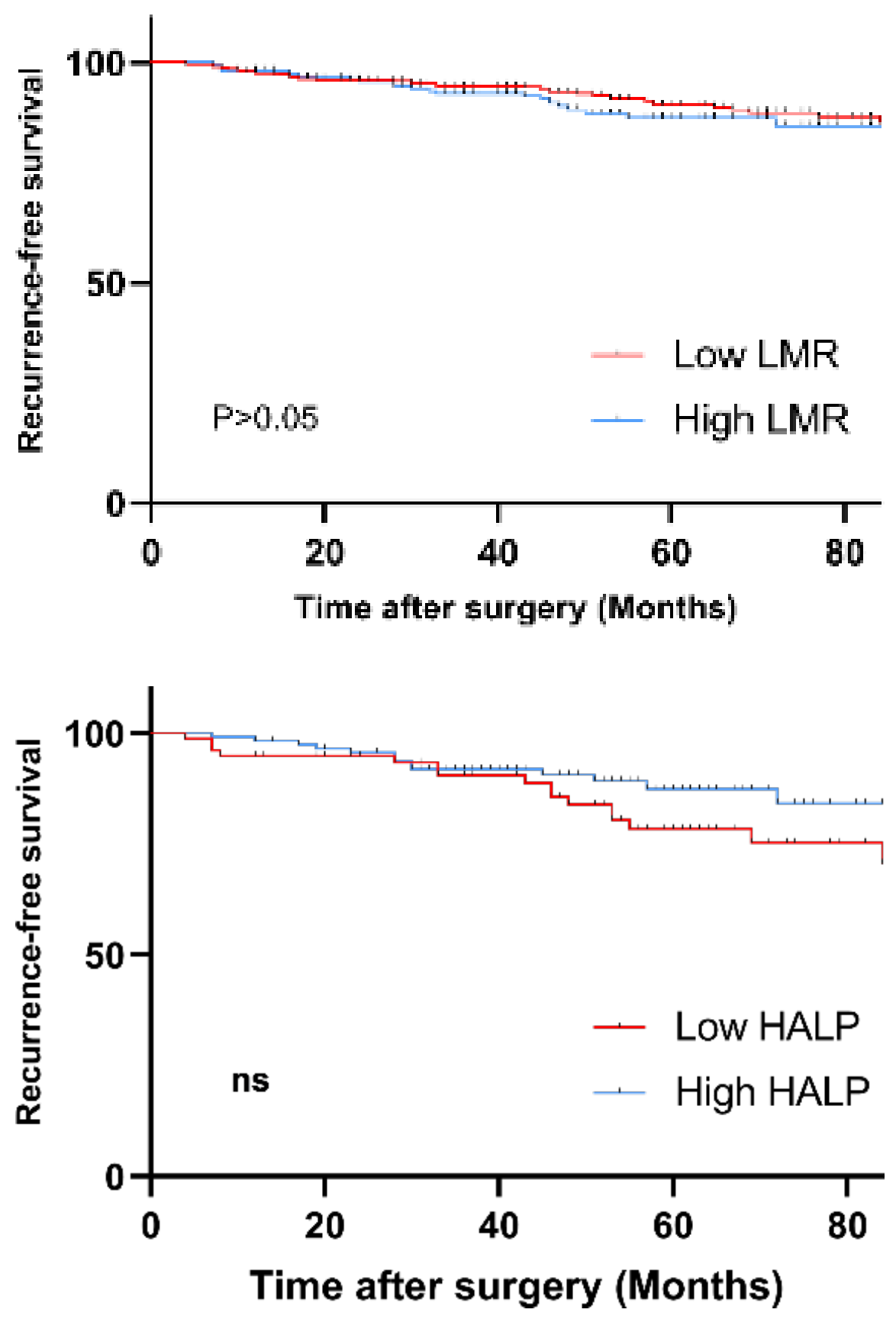

\section{REFERENCE}

1. Ma GL, Murphy JD, Martinez ME, Sicklick JK. Epidemiology of gastrointestinal stromal tumors in the era of histology codes: results of a population-based study. Cancer Epidemiol Biomarkers Prev.2015;24(1):298302 .

2. Nishida T, Blay JY, Hirota S, Kitagawa Y, Kang YK. The standard diagnosis, treatment, and follow-up of gastrointestinal stromal tumors based on guidelines. Gastric Cancer. 2016;19(1):3-14.

3. Barrios CH, Blackstein ME, Blay JY, et al. The GOLD ReGISTry: a Global, Prospective, Observational Registry Collecting Longitudinal Data on Patients with Advanced and Localised Gastrointestinal Stromal 
Tumours. Eur J Cancer. 2015;51(16):2423-2433.

4. DeMatteo RP, Ballman KV, Antonescu CR, et al. Long-term results of adjuvant imatinib mesylate in localized, high-risk, primary gastrointestinal stromal tumor: ACOSOG Z9000 (Alliance) intergroup phase 2 trial. Ann Surg. 2013;258(3):422-429.

5. Demetri GD, von Mehren M, Antonescu CR, et al. NCCN Task Force report: update on the management of patients with gastrointestinal stromal tumors. J Natl Compr Canc Netw. 2010;8 Suppl 2(0 2):S1-41; quiz S42-44.

6. Casali PG, Abecassis N, Aro HT, et al. Gastrointestinal stromal tumours: ESMO-EURACAN Clinical Practice Guidelines for diagnosis, treatment and follow-up. Ann Oncol. 2018;29(Suppl 4):iv68-iv78.

7. Koo DH, Ryu MH, Kim KM, et al. Asian Consensus Guidelines for the Diagnosis and Management of Gastrointestinal Stromal Tumor. Cancer Res Treat. 2016;48(4):1155-1166.

8. Ubukata H, Motohashi G, Tabuchi T, Nagata H, Konishi S, Tabuchi T. Evaluations of interferon$\gamma /$ interleukin-4 ratio and neutrophil/lymphocyte ratio as prognostic indicators in gastric cancer patients. J Surg Oncol. 2010;102(7):742-747.

9. Malietzis G, Giacometti M, Askari A, et al. A preoperative neutrophil to lymphocyte ratio of 3 predicts disease-free survival after curative elective colorectal cancer surgery. Ann Surg.2014;260(2):287-292.

10. Pinato DJ, Shiner RJ, Seckl MJ, Stebbing J, Sharma R, Mauri FA. Prognostic performance of inflammation-based prognostic indices in primary operable non-small cell lung cancer. $B r J$ Cancer.2014;110(8):1930-1935.

11. Sun J, Mei Y, Zhu Q, et al. Relationship of prognostic nutritional index with prognosis of gastrointestinal stromal tumors. J Cancer. 2019;10(12):2679-2686.

12. Shi WK, Zhang XH, Zhang J, et al. Predictive ability of prognostic nutritional index in surgically resected gastrointestinal stromal tumors: a propensity score matching analysis. Jpn J Clin Oncol.2019;49(9):823-831.

13. Yin XN, Tang SM, Yin Y, Shen CY, Zhang B, Chen ZX. [Associations of Preoperative Platelet-tolymphocyte Ratio and Derived Neutrophil-to-lymphocyte Ratio with thePrognosis of Gastrointestinal Stromal Tumor]. Sichuan Da Xue Xue Bao Yi Xue Ban.2017;48(2):239-243.

14. Feng F, Tian Y, Liu S, et al. Combination of PLR, MLR, MWR, and Tumor Size Could Significantly Increase the Prognostic Value for Gastrointestinal Stromal Tumors. Medicine (Baltimore).2016;95(14):e3248.

15. Yin Z, Gao J, Liu W, et al. Clinicopathological and Prognostic Analysis of Primary Gastrointestinal Stromal Tumor Presenting with Gastrointestinal Bleeding: a 10-Year Retrospective Study. J Gastrointest Surg. 2017;21(5):792-800.

16. Racz JM, Cleghorn MC, Jimenez MC, et al. Predictive Ability of Blood Neutrophil-to-Lymphocyte and Platelet-to-Lymphocyte Ratios in Gastrointestinal Stromal Tumors. Ann Surg Oncol.2015;22(7):2343-2350.

17. Luo XF, Zhou LH. Prognostic significance of neutrophil to lymphocyte ratio in patients with gastrointestinal stromal tumors: A meta-analysis. Clin Chim Acta. 2018;477:7-12.

18. Chen XL, Xue L, Wang W, et al. Prognostic significance of the combination of preoperative hemoglobin, albumin, lymphocyte and platelet in patients with gastric carcinoma: a retrospective cohort study. Oncotarget. 2015;6(38):41370-41382.

19. Xu SS, Li S, Xu HX, et al. Haemoglobin, albumin, lymphocyte and platelet predicts postoperative survival in pancreatic cancer. World J Gastroenterol. 2020;26(8):828-838.

20. Peng D, Zhang CJ, Tang Q, et al. Prognostic significance of the combination of preoperative hemoglobin and albumin levels and lymphocyte and platelet counts (HALP) in patients with renal cell carcinoma after nephrectomy. BMC Urol. 2018;18(1):20. 
21. Guo Y, Shi D, Zhang J, et al. The Hemoglobin, Albumin, Lymphocyte, and Platelet (HALP) Score is a Novel Significant Prognostic Factor for Patients with Metastatic Prostate Cancer Undergoing Cytoreductive Radical Prostatectomy. J Cancer. 2019;10(1):81-91.

22. Peng D, Zhang CJ, Gong YQ, et al. Prognostic significance of HALP (hemoglobin, albumin, lymphocyte and platelet) in patients with bladder cancer after radical cystectomy. Sci Rep. 2018;8(1):794.

23. Cong $\mathrm{L}, \mathrm{Hu} \mathrm{L}$. The value of the combination of hemoglobin, albumin, lymphocyte and platelet in predicting platinum-based chemoradiotherapy response in male patients with esophageal squamous cell carcinoma.Int Immunopharmacol. 2017;46:75-79.

24. Shen XB, Zhang YX, Wang W, Pan YY. The Hemoglobin, Albumin, Lymphocyte, and Platelet (HALP) Score in Patients with Small Cell Lung Cancer Before First-Line Treatment with Etoposide and ProgressionFree Survival. Med Sci Monit. 2019;25:5630-5639.

25. Ichikawa K, Mizuno S, Hayasaki A, et al. Prognostic Nutritional Index After Chemoradiotherapy Was the Strongest Prognostic Predictor Among Biological and Conditional Factors in Localized Pancreatic Ductal Adenocarcinoma Patients. Cancers (Basel). 2019;11(4).

26. Hsu JT, Wang CC, Le PH, et al. Lymphocyte-to-monocyte ratios predict gastric cancer surgical outcomes. J Surg Res.2016;202(2):284-290.

27. Joensuu H. Risk stratification of patients diagnosed with gastrointestinal stromal tumor. Hum Pathol.2008;39(10):1411-1419.

28. Camp RL, Dolled-Filhart M, Rimm DL. X-tile: a new bio-informatics tool for biomarker assessment and outcome-based cut-point optimization. Clin Cancer Res. 2004;10(21):7252-7259.

29. Heagerty PJ, Lumley T, Pepe MS. Time-dependent ROC curves for censored survival data and a diagnostic marker. Biometrics.2000;56(2):337-344.

30. Goh BK, Chok AY, Allen JC, Jr., et al. Blood neutrophil-to-lymphocyte and platelet-to-lymphocyte ratios are independent prognostic factors for surgically resected gastrointestinal stromal tumors. Surgery. $2016 ; 159(4): 1146-1156$.

31. Rutkowski P, Teterycz P, Klimczak A, Bylina E, Szamotulska K, Lugowska I. Blood neutrophil-tolymphocyte ratio is associated with prognosis in advanced gastrointestinal stromal tumors treated with imatinib. Tumori. 2018;104(6):415-422.

32. Sorour MA, Kassem MI, Ghazal Ael H, El-Riwini MT, Abu Nasr A. Gastrointestinal stromal tumors (GIST) related emergencies. Int J Surg. 2014;12(4):269-280.

33. Ostroumov D, Fekete-Drimusz N, Saborowski M, Kühnel F, Woller N. CD4 and CD8 T lymphocyte interplay in controlling tumor growth. Cell Mol Life Sci. 2018;75(4):689-713.

34. Shankaran V, Ikeda H, Bruce AT, et al. IFNgamma and lymphocytes prevent primary tumour development and shape tumour immunogenicity.Nature. 2001;410(6832):1107-1111.

35. Thorsson V, Gibbs DL, Brown SD, et al. The Immune Landscape of Cancer. Immunity. 2018;48(4):812830.e814.

36. Nash GF, Turner LF, Scully MF, Kakkar AK. Platelets and cancer.Lancet Oncol. 2002;3(7):425-430.

37. Haemmerle M, Stone RL, Menter DG, Afshar-Kharghan V, Sood AK. The Platelet Lifeline to Cancer: Challenges and Opportunities. Cancer Cell. 2018;33(6):965-983.

38. Davì G, Patrono C. Platelet activation and atherothrombosis. N Engl J Med. 2007;357(24):2482-2494.

39. Abdol Razak NB, Jones G, Bhandari M, Berndt MC, Metharom P. Cancer-Associated Thrombosis: An Overview of Mechanisms, Risk Factors, and Treatment. Cancers (Basel). 2018;10(10). 
40. Olsson AK, Cedervall J. The pro-inflammatory role of platelets in cancer. Platelets. 2018;29(6):569-573. 41. Schlesinger M. Role of platelets and platelet receptors in cancer metastasis. J Hematol Oncol. 2018;11(1):125.

42. Wojtukiewicz MZ, Sierko E, Hempel D, Tucker SC, Honn KV. Platelets and cancer angiogenesis nexus. Cancer Metastasis Rev.2017;36(2):249-262.

43. Yang Z, Wang F, Liu S, Guan W. Comparative clinical features and short-term outcomes of gastric and small intestinal gastrointestinal stromal tumours: a retrospective study. Sci Rep. 2019;9(1):10033.

44. Italiano A, Cioffi A, Coco P, et al. Patterns of care, prognosis, and survival in patients with metastatic gastrointestinal stromal tumors (GIST) refractory to first-line imatinib and second-line sunitinib.Ann Surg Oncol. 2012;19(5):1551-1559.

45. Hompland I, Bruland Ø S, Hølmebakk T, et al. Prediction of long-term survival in patients with metastatic gastrointestinal stromal tumor: analysis of a large, single-institution cohort. Acta Oncol.2017;56(10):13171323.

46. Xia Y, Chen S, Luo M, et al. Correlations between imatinib plasma trough concentration and adverse reactions in Chinese patients with gastrointestinal stromal tumors. Cancer. 2020;126 Suppl 9:2054-2061.

47. Peng B, Lloyd P, Schran H. Clinical pharmacokinetics of imatinib.Clin Pharmacokinet. 2005;44(9):879894.

48. Allen TM, Cullis PR. Drug delivery systems: entering the mainstream.Science. 2004;303(5665):1818-1822.

49. Kalyane D, Raval N, Maheshwari R, Tambe V, Kalia K, Tekade RK. Employment of enhanced permeability and retention effect (EPR): Nanoparticle-based precision tools for targeting of therapeutic and diagnostic agent in cancer. Mater Sci Eng C Mater Biol Appl.2019;98:1252-1276.

50. Kim B, Seo B, Park S, et al. Albumin nanoparticles with synergistic antitumor efficacy against metastatic lung cancers. Colloids Surf B Biointerfaces. 2017;158:157-166.

51. Cohen MH, Cortazar P, Justice R, Pazdur R. Approval summary: imatinib mesylate in the adjuvant treatment of malignant gastrointestinal stromal tumors. Oncologist. 2010;15(3):300-307.

\section{Hosted file}

figure 1.docx available at https://authorea.com/users/420685/articles/526972-combined-indexof-hemoglobin-albumin-lymphocyte-and-platelet-halp-predict-recurrence-after-radicalresection-in-gastrointestinal-stromal-tumors-a-propensity-scores-matching-analysis

\section{Hosted file}

figure 2.docx available at https://authorea.com/users/420685/articles/526972-combined-indexof-hemoglobin-albumin-lymphocyte-and-platelet-halp-predict-recurrence-after-radicalresection-in-gastrointestinal-stromal-tumors-a-propensity-scores-matching-analysis

\section{Hosted file}

figure 3.docx available at https://authorea.com/users/420685/articles/526972-combined-indexof-hemoglobin-albumin-lymphocyte-and-platelet-halp-predict-recurrence-after-radicalresection-in-gastrointestinal-stromal-tumors-a-propensity-scores-matching-analysis

\section{Hosted file}

Table 1.docx available at https://authorea.com/users/420685/articles/526972-combined-indexof-hemoglobin-albumin-lymphocyte-and-platelet-halp-predict-recurrence-after-radicalresection-in-gastrointestinal-stromal-tumors-a-propensity-scores-matching-analysis 


\section{Hosted file}

Table 2.docx available at https://authorea.com/users/420685/articles/526972-combined-indexof-hemoglobin-albumin-lymphocyte-and-platelet-halp-predict-recurrence-after-radicalresection-in-gastrointestinal-stromal-tumors-a-propensity-scores-matching-analysis

\section{Hosted file}

Table S2.docx available at https://authorea.com/users/420685/articles/526972-combined-indexof-hemoglobin-albumin-lymphocyte-and-platelet-halp-predict-recurrence-after-radicalresection-in-gastrointestinal-stromal-tumors-a-propensity-scores-matching-analysis 